\title{
Double magnetocaloric peak feature observed in quaternary Ni-Mn-In based Heusler alloys
}

\author{
I. D. Rodionov ${ }^{1, \star}$, I. S. Dubenko ${ }^{2,}$, V. N. Prudnikov ${ }^{1}$, A. B. Granovsky ${ }^{1}$, I. S. Titov ${ }^{1,3}$, and A. M. Saletsky ${ }^{1}$ \\ ${ }^{1}$ Faculty of Physics, Lomonosov Moscow State University, Moscow, Russia \\ ${ }^{2}$ Department of Physics, Southern Illinois University, Carbondale, USA \\ ${ }^{3}$ Physics and Materials Science Research Unit, University of Luxembourg, Luxembourg, Luxembourg
}

\begin{abstract}
We present the results of the studies of magnetic and magnetocaloric (MCE) properties of quaternary Heusler alloys $\mathrm{Ni}_{48.5} \mathrm{Mn}_{35} \mathrm{In}_{15} \mathrm{Co}_{1.5}$ and $\mathrm{Ni}_{50} \mathrm{Mn}_{35} \mathrm{In}_{13.5} \mathrm{Al}_{1.5}$ in temperature range between $80 \mathrm{~K}$ and 400 K. While doping initial ternary Ni-Mn-In alloy with 1.5 at.\% Al does not change $T_{C}^{A}$ as well as $M_{S}$, doping with 1.5 at.\% Co significantly increases both $T_{C}^{A}$ and $M_{S}$. The direct magnetocaloric measurements around martensitic transition and austenite Curie temperature reveal the competition between the contributions of different signs. The latter is related to the vicinity of transition temperatures of austenite and martensite, resulting in mixed state, where both phases undergo magnetic order-disorder transformation in the same temperature region. Additionally temperature dependencies of adiabatic temperature change $\Delta T_{a d}$ and magnetic entropy $\Delta S_{M}$ of the system exhibit additional peak feature which we attribute to intermartensitic transition occurring in these alloys.
\end{abstract}

\section{Introduction}

Study of magnetocaloric effect (MCE) in different multifunctional materials is very attractive due to huge potential of it's practical applications [1]. Using magnetocaloric material as working body of magnetic refrigerator [2] leads to significant decrease of harmful waste release, reduction of consumed electricity and increasing of efficiency up to $30 \%$ [3]. Additionally, MCE may be used in medicine for destruction of caner cells applying hyperthermia treatment $[4,5]$. Heusler alloys are one of the most promising MCE materials with large amount of $\Delta T_{a d}$ at applied magnetic field [6, 7].

Off-stoichiometric Heusler alloys based on Ni-Mn-In undergo martensitic transition (MT) - structural diffusionless transition from high temperature austenite $L 2_{1}$ phase to low temperature $L 1_{0}$ phase. This transition reveals strong dependence between structural and magnetic subsystems - MT may be induced both by temperature change or by applying magnetic field. Doping by fourth element in low concentration (no more then 5 at.\%) one can drastically change martensitic transition parameters (martensite start $M_{S}$ and finish temperatures $M_{F}$, width of temperature hysteresis, saturation magnetization per unit cell in martensite and austenite states) [6].

MT proceeds with increase of volume of unit cell. Due to that reason inner mechanical stress appears and it may accompanied by formation of modulated structures [8-10]. Lowering temperature modulated structure may transform into non-modulated martensite phase and such

\footnotetext{
^e-mail: rodionov@physics.msu.ru
}

transformation is called intermartensitic transition (IMT). In general IMT means sequence of phase transitions between modulated and non-modulated states of martensite phase. Similarly to MT, the presence of IMT may be detected by several methods. The most reliable way to find the IMT it is XRD analysis in vicinity of phase transition temperature range [11]. The temperature dependence of magnetization exhibits step-like behavior at hysteresis loop [12]. IMT also can be found from temperature dependencies of resistivity [11].

In this publication we present the results of magnetic and MCE study of quaternary Ni-Mn-In based Heusler alloys doped by $\mathrm{Al}$ and $\mathrm{Co}$ which reveal step-like behavior of magnetization, $\Delta T_{a d}(T)$ and $\Delta S_{M}(T)$ in vicinity of MT.

\section{Experiment}

Polycrystalline bulk samples were prepared by arc melting $4 \mathrm{~N}$ purity metals under argon atmosphere followed by annealing in high vacuum $\left(\sim 10^{-5}\right.$ Torr) for $24 \mathrm{~h}$ at $1123 \mathrm{~K}$. The chemical composition of as-prepared samples was measured by energy-dispersive X-ray spectroscopy (EDS) and its relative deviations were less than 1 at.\%. The room temperature structural analysis was carried out with conventional x-ray diffraction. Magnetic measurements were carried out with VSM Lake Shore in ZFC and FC regimes in a temperature range between $80-400 \mathrm{~K}$ in high $(1.6 \mathrm{~T})$ and low $(5 \mathrm{mT})$ magnetic fields. Indirect determination of the magnetic entropy change $\Delta S_{M}$ were performed by calculation of $\Delta S_{M}$ via Maxwell equation from isothermal 
magnetization curves $M(H)$ :

$$
\Delta S_{\mathrm{M}}(T, \Delta H)=\int_{0}^{H} \frac{\partial M(T, H)}{\partial T} d H
$$

Direct measurements of the adiabatic temperature change $\Delta T_{a d}$ in changing field $\mu_{0} \Delta H=1.8 \mathrm{~T}$ were carried out using an adiabatic magnetocalorimeter $(\mathrm{MagEq}$ MMS 801) in a temperature range between $250-350 \mathrm{~K}$.

\section{Results and discussion}

Temperature dependencies of magnetization at low and high magnetic fields for $\mathrm{Ni}_{50} \mathrm{Mn}_{35} \mathrm{In}_{13.5} \mathrm{Al}_{1.5}$ are presented on fig. 1 (a) and (c). According this data the sample undergoes magnetic order-disorder transition at $T_{C}^{A}=324 \mathrm{~K}$. Cooling to $M_{S}=294 \mathrm{~K}$ starts forward MT which finishes at $M_{F}=250 \mathrm{~K}$. Abrupt change of magnetization between two phases are equal to $\Delta M=33 \mathrm{emu} / \mathrm{g}$ at $H=1.6 \mathrm{~T}$. For $\mathrm{Ni}_{48.5} \mathrm{Mn}_{35} \mathrm{In}_{15} \mathrm{Co}_{1.5} M(T)$ dependencies are presented on fig. 1 (b) and (d). Magnetic transition from ferromagnetic to paramagnetic state occurs at $T_{C}^{A}=360 \mathrm{~K}$. MT start and finish temperatures are respectively $M_{S}=342 \mathrm{~K}$ and $M_{F}=308 \mathrm{~K}$. Magnetization drop during MT $\Delta M=$ $54 \mathrm{emu} / \mathrm{g}$. Low temperature hysteresis was detected between $80 \mathrm{~K}$ and $140 \mathrm{~K}$ (fig. 1 (b)). The width of this hysteresis increases with increasing of external magnetic field.

It is worth mentioning that magnetization in area of martensitic transition reveals step-like $M(T)$ dependence on cooling and heating both in case of $\mathrm{Al}$ and Co-doped alloys. For Co-doped alloys such behavior clearly seen in vicinity of $M_{I M}=338 \mathrm{~K}$ (fig. 1 (b)). Also such non monotonous behavior of magnetization take place in case of $M(T)$ plotted from $M(H)$ data in wide magnetic field range from $5 \mathrm{~m} \mathrm{~T}$ to $1.6 \mathrm{~T}$.
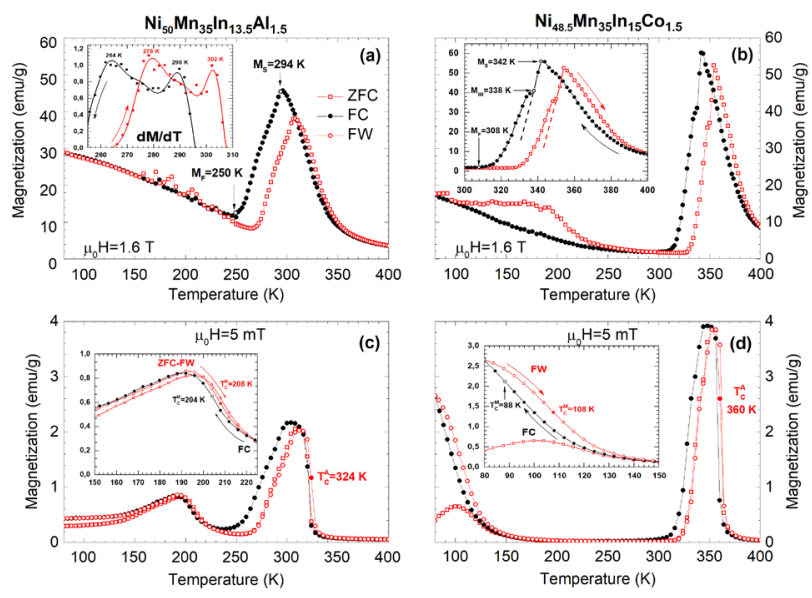

Figure 1. Temperature dependencies of magnetization for $\mathrm{Ni}_{50} \mathrm{Mn}_{35} \mathrm{In}_{13.5} \mathrm{Al}_{1.5}$ and $\mathrm{Ni}_{48.5} \mathrm{Mn}_{35} \mathrm{In}_{15} \mathrm{Co}_{1.5}$ samples measured in $1.6 \mathrm{~T}((\mathrm{a})$ and (b)) and $5 \mathrm{mT}((\mathrm{c})$ and (d)), respectively.

Fig. 2 (a) and (b) show temperature dependencies of adiabatic temperature change $\Delta T_{a d}$ on applying $1.8 \mathrm{~T}$ for

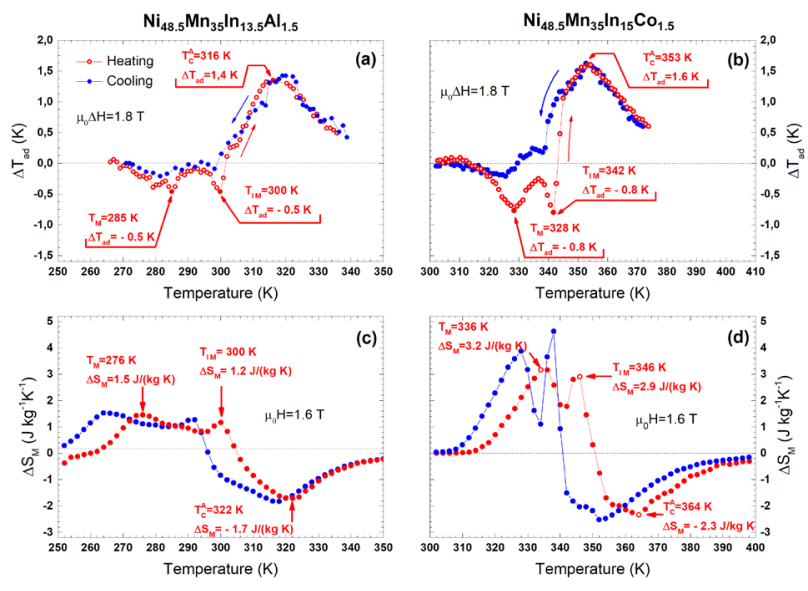

Figure 2. Temperature dependencies of adiabatic temperature change on applying $1.8 \mathrm{~T} \Delta T_{a d}$ and magnetic entropy change $\Delta S_{M}$ in $1.6 \mathrm{~T}$ around the martensitic transition for $\mathrm{Ni}_{50} \mathrm{Mn}_{35} \operatorname{In}_{13.5} \mathrm{Al}_{1.5}$ and $\mathrm{Ni}_{48.5} \mathrm{Mn}_{35} \mathrm{In}_{15} \mathrm{Co}_{1.5}$ samples ((a) and (c)) and ((b) and (d)), respectively. Characteristic transition temperatures are marked with arrows.

$\mathrm{Ni}_{50} \mathrm{Mn}_{35} \operatorname{In}_{13.5} \mathrm{Al}_{1.5}$ and $\mathrm{Ni}_{48.5} \mathrm{Mn}_{35} \mathrm{In}_{15} \mathrm{Co}_{1.5}$ samples, respectively. Both samples exhibit maximum of $\Delta T_{a d}$ of about $1.5 \mathrm{~K}$ in the vicinity of austenite Curie temperature $T_{C}^{A}: \mathrm{Ni}_{50} \mathrm{Mn}_{35} \mathrm{In}_{13.5} \mathrm{Al}_{1.5}$ at $316 \mathrm{~K}$ and $\mathrm{Ni}_{48.5} \mathrm{Mn}_{35} \mathrm{In}_{15} \mathrm{Co}_{1.5}$ at $353 \mathrm{~K}$, respectively. Al-doped sample shows two inverse MCE peaks in the region of the martensitic transition on heating: at $T_{M}=285 \mathrm{~K} 26 \mathrm{~K}$ wide and at $T_{I M}=300 \mathrm{~K}$ $7 \mathrm{~K}$ wide. Both peaks show the same magnitude of about $0.5 \mathrm{~K}$. Co-doped sample also shows similar $\Delta T_{a d}$ dependence on heating with double peak feature: at $T_{M}=328 \mathrm{~K}$ $6 \mathrm{~K}$ wide and at $T_{I M}=342 \mathrm{~K} 24 \mathrm{~K}$ wide. Both peaks show the same magnitude of about $0.8 \mathrm{~K}$.

Fig. 2 (c) and (d) show temperature dependencies of magnetic contribution to total entropy of the system $\Delta S_{M}$ for $\mathrm{Ni}_{50} \mathrm{Mn}_{35} \mathrm{In}_{13.5} \mathrm{Al}_{1.5}$ and $\mathrm{Ni}_{48.5} \mathrm{Mn}_{35} \mathrm{In}_{15} \mathrm{Co}_{1.5}$ samples, respectively. $\Delta S_{M}$ was calculated following the Maxwell equation (see eq. 1) from the series of isothermal magnetization curves measured on cooling and heating with step of $2 \mathrm{~K}$ in temperature with subsequent return martensite (austenite) state on heating (cooling).

For the Al-doped sample the maximum entropy change $\left|\Delta S_{M}\right|=1.7 \mathrm{~J} / \mathrm{kg} \mathrm{K}$ is observed around $T_{C}^{A}=322 \mathrm{~K}$. In the region of the martensitic transition similar double peak feature is present: $\left|\Delta S_{M}\right|=1.5 \mathrm{~J} / \mathrm{kg} \mathrm{K}$ at $T_{M}=276 \mathrm{~K}$ and $\left|\Delta S_{M}\right|=1.2 \mathrm{~J} / \mathrm{kg} \mathrm{K}$ at $T_{I M}=300 \mathrm{~K}$. For the Co-doped sample the maximum entropy change $\left|\Delta S_{M}\right|=2.3 \mathrm{~J} / \mathrm{kg} \mathrm{K}$ is observed around $T_{C}^{A}=364 \mathrm{~K}$, two peaks are present during martensitic transition: $\left|\Delta S_{M}\right|=3.2 \mathrm{~J} / \mathrm{kg} \mathrm{K}$ at $T_{M}=$ $336 \mathrm{~K}$ and $\left|\Delta S_{M}\right|=2.9 \mathrm{~J} / \mathrm{kg} \mathrm{K}$ at $T_{I M}=346 \mathrm{~K}$. The difference between $\Delta S_{M}(T)$ curves on heating and cooling for Co-doped sample comes into agreement with $M(T)$ for this sample.

In summary, doping initial ternary $\mathrm{Ni}_{50} \mathrm{Mn}_{35} \operatorname{In}_{15}$ alloy with 1.5 at.\% $\mathrm{Al}$ does not change $T_{C}^{A}$ as well as $M_{S}$, doping with 1.5 at.\% Co significantly increases both $T_{C}^{A}$ and $M_{S}[13]$. However, both Al- and Co-doped samples 
exhibit step-like behavior in temperature dependence of magnetization $M(T)$ as well as double peak feature in both magnetic entropy change $\Delta S_{M}$ and adiabatic temperature change $\Delta T_{a d}$, these features are not observed in initial ternary alloy. One of the reasons for this peculiar behavior could be the intermartensitic transformation happening during the conventional martensitic transition. Such transitions between $L 1_{0}$ and modulated martensite phases were reported by several groups, see[6,12]. The origin and features of such behavior will be the subject of further temperature-dependent $\mathrm{x}$-ray diffraction and differential scanning calorimetry studies.

We acknowledge financial support from the Russian Foundation for Basic Research (RFBR): grant No. 15-02$01976 \mathrm{~A}$ and 16-32-00460 mol a.

\section{References}

[1] O. Gutfleisch et al., Advanced Materials 23 (7), p. 841-842 (2011)

[2] K. Gschneidner et al., International Journal of Refrigeration 31 (6), pp. 945-961 (2008)
[3] C. Zimm et al., Advances in Cryogenic Engineering 43, pp. 1759-1766 (1998)

[4] A. M. Tishin et al., Recent Patents on Anti-Cancer Drug Discovery 11 (4), pp. 360-375 (2016)

[5] A. M. Tishin et al., International Journal of Refrigeration 68, pp. 177-186 (2016)

[6] J. Liu et al., Nature Materials 11 (7), p. 620-6 (2012)

[7] A. Quetz et al., Journal of Alloys and Compounds 683, p. 139-142 (2016)

[8] K. Otsuka et al., Scripta Metallurgica et Materiala 29 (10), pp. 1359-1364 (1993)

[9] P. J. Brown et al., Journal of Physics: Condensed Matter 18 (7), p. 2249 (2006)

[10] S. Kaufmann et al., Physical Review Letters 104 (14) (2010)

[11] A.Cakir et al., Journal of Applied Physics 114 (18) (2013)

[12] S. Y. Dong et al., Scientific Reports 6 (2016)

[13] I. D. Rodionov et al., JETP Letters 311 (6), pp. 385-389 (2015) 